\title{
Transformations between Vilnius and Strömgren photometric systems ${ }^{\star}$
}

\author{
N. Kaltcheva ${ }^{1}$ and J. Knude ${ }^{2}$ \\ 1 Department of Physics \& Astronomy, University of Wisconsin Oshkosh, 800 Algoma Blvd., Oshkosh, \\ WI 54901-8644, USA \\ 2 Niels Bohr Institute for Astronomy, Physics and Geophysics, Juliane Maries Vej 30, 2100 Copenhagen $\varnothing$, \\ Denmark \\ e-mail: indus@astro.ku.dk
}

Received 13 June 2001 / Accepted 17 January 2002

\begin{abstract}
We present a number of relationships between similar colour indices in the Vilnius and Strömgren photometric systems, empirically derived from all observational data available. Significant differences between the corresponding indices, apparently due to the different central wavelength and width of the Strömgren $u$ and $v$ bands and Vilnius $U$ and $X$ bands are found. We demonstrate that the effects may depend on metallicity and luminosity. For G-K stars of moderate luminosity we interpret the difference introduced by $v$ and $X$ passbands as an $\log g$ effect, reflecting the presence of Ca II lines in these spectral types. Apart from the interstellar Ca II absorption, the difference $(v-X)$ is little affected by the interstellar dust. It turns out as well to be very straightforward to make an estimation of the $[\mathrm{Fe} / \mathrm{H}]$ index with an accuracy of 0.4 dex, using the Vilnius $U$ and Strömgren $u$ filters. A comparison of $(v-X)$ to the $h k$ index is also presented, supporting the conclusion that $X$ magnitude is strongly influenced by the Ca II lines. We discuss a possible influence of the Ca II stellar lines on the absolute magnitude inferred from the Vilnius photometry.
\end{abstract}

Key words. methods: observational - techniques: photometric - photometric systems: Strömgren, Vilnius, Strömvil

\section{Introduction}

The importance of both Strömgren and Vilnius photometry has been recently emphasized with the introduction of their combination the Strömvil system (Straizys et al. 1996a). The mean wavelengths of the uvby bands are close to the $U X Y V$ magnitudes in the Vilnius system. In addition, the Vilnius system contains another three passbands $P Z S$, which are introduced for classification of latetype stars and for detection of stellar emission. This makes it possible to create a combined system uvbyP $Z S$, which is capable to give a two-dimensional stellar classification in presence of variable interstellar reddening, preserving at the same time a possibility to solely apply the well established $u v b y$ calibrations. Investigating the relationships between corresponding colour indices in the Vilnius and Strömgren photometric systems is helpful to understand the effects of luminosity and metallicity that affect the photometric quantities for different spectral types, and to study the possibility of their mutual replacement. This will also cast light on the combined characteristics of the

Send offprint requests to: N. Kaltcheva,

e-mail: kaltchev@uwosh.edu

* Based on CDS data.
Strömvil system, and will eventually allow a transformation between the existing data catalogs. Furthermore, this will facilitate the calibration of the Strömvil photometry in terms of fundamental stellar parameters. In particular we may investigate whether the Strömvil system reproduces results obtained with the uvby $\beta$ photometry.

The correlations between magnitudes and colours of different photometric systems are complex and depend on the physical parameters of the stars and their reddening. A computation of such relationships, including Strömgren and Vilnius indices, has been presented by Straizys (1973), based on theoretical integration of the energy distribution curves in stellar spectra, the response curves and the curve of transmission by interstellar matter. Recently Straizys et al. (1996a) investigated the interrelationships between similar magnitudes in the Strömgren and Vilnius systems as a function of $B-V$ colour index, based on synthetic colours. In the present paper we apply an entirely empirical approach, based on the published photometric data in the Strömgren and Vilnius photometric systems.

\section{The sample}

The sample used in the present study is created in the following way. All stars with both Vilnius and Strömgren full or partial photometric data were extracted using the 
VizieR II/157A (Photoelectric Photometry in Vilnius system, Straizys + 1989) and VizieR II/215 (uvby $\beta$ Catalog, Hauck + 1997) catalogs. All multiple stars marked with "AB", "A", "B", "C", etc. flags in the above catalogs were excluded. The overlap consists of nearly 1600 stars.

The spectral classification adopted in the present paper was taken from the II/157A catalog. The main sources of spectral classification used there are the catalogs by M. Jaschek (Selected Spectral Types in the MK system (CDS Catalogue: III/42) =1978BICDS 15, 121), by P. M. Kennedy (MK Classification Extension Catalog, Mount Stromlo, Australia, ed. 1978), the Bright Star Catalog, GCVS and General Catalog of Cool Carbon Stars of C. B. Stephenson (Publ. Warner and Swasey Obs., Vol. 1, No. 4, 1973). If a classification based on spectra is not available, classification obtained from the Vilnius photometry is adopted in the II/157A catalog. In the present study however, we excluded from our sample all stars without a spectra-based classification. We also excluded all stars that show differences in the $V$ magnitudes listed in the Vilnius and Strömgren catalogs larger than $\pm 0.02 \mathrm{mag}$, to secure against variability or mis-identification. The remaining stars are separated in sub-samples of $\mathrm{O}, \mathrm{A}, \mathrm{F}, \mathrm{G}$, $\mathrm{K}$ and $\mathrm{M}$ types, according to the available spectral classification. Each sub-sample contains all ranges of luminosity classes from V to Ia, including stars with peculiarities mentioned in the spectra as well. Only the sub-dwarfs F, $\mathrm{G}$ and $\mathrm{K}$ type are excluded.

We compared the MK classification for the stars in all sub-samples to the photometric classification that may be derived from the Strömgren and Vilnius photometry. The quantities $\left[c_{1}\right]=c_{1}-0.20(b-y)$ and $\left[m_{1}\right]=m_{1}+$ $0.32(b-y)$ were calculated and the $\left[c_{1}\right] /\left[m_{1}\right]$ diagrams for the different sub-samples are presented in Fig. 1. The main-sequence is obtained based on the intrinsic colour relations by Philip \& Egret (1980). The $\left[c_{1}\right] /\left[m_{1}\right]$ diagram is essential for refining the spectral classification in the Strömgren systems (Strömgren 1966; Olsen 1983). The diagrams in Fig. 1 were visually compared to the classification diagram presented by Strömgren (1966, his Fig. 1). In general, there is a close correspondence between the spectral classification available in the literature and the photometric classification based on the $\left[c_{1}\right] /\left[m_{1}\right]$ diagrams for $\mathrm{O}, \mathrm{B}, \mathrm{A}$ and $\mathrm{F}$ spectral types. As far as the G type subsample is concerned, a systematic mis-classifications as $\mathrm{F}$ and $\mathrm{K}$ types may exist.

Then we calculated the indices $Q_{\mathrm{X} Y V}=(X-Y)-$ $E_{\mathrm{XY}} / E_{\mathrm{YV}}(Y-V)$ which are used for preliminary spectral type classification in the Vilnius system. The colour-excess ratios $E_{\mathrm{XY}} / E_{\mathrm{Y} V}$ listed in Table 3 of Straizys et al. (1996) were used in the calculation of $Q_{\mathrm{X} Y V}$ indices $(0.75$ for spectral type B, 0.73 for spectral type A, F, G and 0.70 for spectral type $\mathrm{K})$. Since spectral type $\mathrm{O}$ is not included in Table 3 of Straizys et al. (1996), the $E_{\mathrm{X} Y} / E_{\mathrm{Y} V}$ value for spectral class B was applied. The stars in each subsample were restricted only to those satisfying the Vilnius criterion for preliminary spectral classification. Applying the Vilnius criterion actually left in the sub-samples only
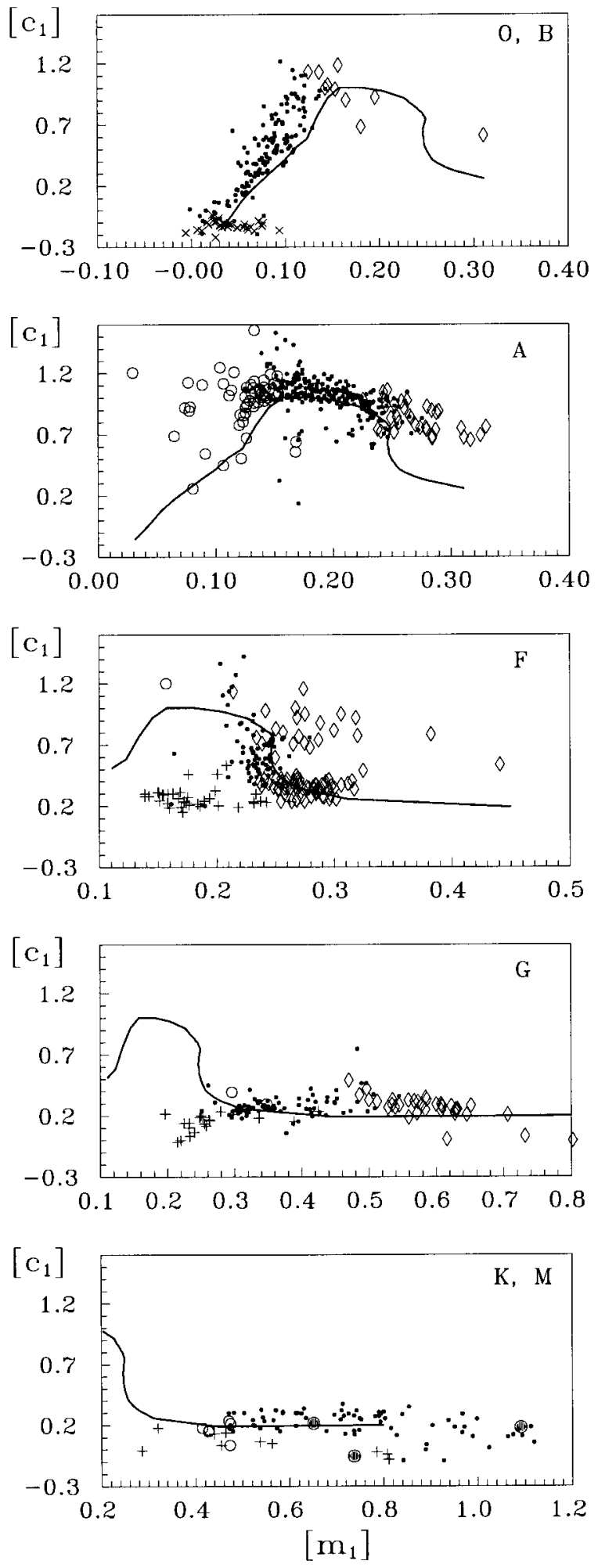

Fig. 1. The diagrams $\left[c_{1}\right] /\left[m_{1}\right]$ for the different sub-samples. Filled symbols are used for the stars that can be referred to the given spectral type sub-sample both according to $Q_{\mathrm{XY} V}$ criterion and $\left[c_{1}\right] /\left[m_{1}\right]$ criterion. Open symbols are used if the $Q_{\mathrm{XYV}}$ value are smaller (open circles) or larger (open diamonds) than the corresponding $Q_{\mathrm{X} Y V}$ range. O, B stars panel - crosses are used for the O-type stars. The position of the F, G, K sub-dwarfs are marked with plus symbols on the corresponding panels. K, M stars panel - the 3 M-type stars in the sample are shown with large filled symbols. 
stars satisfying the Strömgren classification criterion as well. The ranges of $Q_{\mathrm{X} Y V}$ values for different spectral types are given in several publications (cf. for example Table 4, Straizys et al. 1996). In Fig. 1 the stars that fall in the range specified for a given spectral type according to the $Q_{\mathrm{X} Y V}$ quantities are marked with filled symbols. Open symbols are used for the stars outside this range open circles if the calculated $Q_{\mathrm{X} Y V}$ value is smaller than that specified in Table 4 (Straizys et al. 1996), and open diamonds if the $Q_{\mathrm{X} Y V}$ value is larger. For B-type stars the $Q_{\mathrm{XY} V}$ criterion and $\left[c_{1}\right] /\left[m_{1}\right]$ criterion lead to the same result. For A, F and G-type the $Q_{\mathrm{XYV}}$ criterion is stronger than the $\left[c_{1}\right] /\left[m_{1}\right]$ criterion and rejects the late sub-types and the metal-rich $\mathrm{A}$ and $\mathrm{F}$ stars. This restricts the B-type sample to 141 stars, as some stars classified as B are apparently of spectral type A0-A2 or even later, according both to the Strömgren and Vilnius classification. There are no B-type stars in our sample with $Q_{\mathrm{X} Y V}$ value smaller than specified for this spectral range. In Fig. 1 the O-type stars are plotted with crosses at the same panel as the B-type stars. All O-type stars have $Q_{\mathrm{X} Y V}$ values within the listed limits and this sub-sample consists of 31 stars.

In the derivation of the interrelationships, we will use only stars that satisfy both criteria. A significant number of stars from the A, F and G sub-samples are outside the specified $Q_{\mathrm{XY} V}$ ranges, and are thus excluded from the calculation of the interrelations. A-type stars with too large $\left[m_{1}\right]$ or $Q_{\mathrm{XYV}}$ may be metal rich and those with too small indices $-\lambda$ Boo candidates. In particular there seems to be a mis-classification of early G-type stars as F stars. And likewise a significant number of spectral type G candidates seem later type than the photometric classification. The restrictions on $Q_{\mathrm{X} Y V}$ values excluded also most of the super-giant $\mathrm{A}, \mathrm{F}$ and $\mathrm{G}$ stars from the following comparison and these sub-samples were reduced to 271 , 84 and 74 stars respectively. It should be mentioned however, that including all stars regardless of the $Q_{\mathrm{X} Y V}$ values in the derivation of the interrelations does not affect the calculated coefficients within the errors. This is owing to the closeness of the pass-bands and to the existence of an unique transformation between the corresponding indices for close spectral types.

There are 71 stars included in our K-type sub-sample. Since only three M-type stars have both Vilnius and Strömgren photometry available, we do not discuss this spectral type hereafter.

\section{Discussion}

As already mentioned, four of the pass-bands of the Vilnius system $(U X Y V)$ (at central wavelengths 345 , $405,466,544 \mathrm{~nm}$ respectively) are close analogs of the uvby pass-bands $(350,411,467,547 \mathrm{~nm})$. Thus both the colour differences $(u-v)$ and $(U-X)$ give the intensity near $350 \mathrm{~nm}$ (the strength of the Balmer discontinuity), which is not blanketing free, as the blanketing at $350 \mathrm{~nm}$ is roughly twice larger than at $410 \mathrm{~nm}$. The indices $(v-b)$ and $(X-Y)$ are measures for the blanketing effects at
$410 \mathrm{~nm}$, thus for the $[\mathrm{Fe} / \mathrm{H}]$ ratio. The indices $b-y$ and $(Y-V)$ are reasonably blanketing free, and thus are temperature indicators. It should be mentioned however, that due to the larger width $22 \mathrm{~nm}$ vs. $19 \mathrm{~nm}$ of the $X$ filter in comparison to the $v$ filter, some strong spectral features are included in it, but not in the Strömgren $v$ filter. The Ca II lines at $394 \mathrm{~nm}$ are at $50 \%$ transmission in $X$ but out of the $v$ filter. Thus we may likely see the Ca II - spectral type variation in $X$ but not in $v$. Another strong spectral feature that affects the $X$ filter, but not the $v$ filter is the He line at $396 \mathrm{~nm}$ in the spectra of $\mathrm{O}$ and early B-type stars.

\subsection{Theoretical relationships}

To establish the transformations between the corresponding colour indices, Straizys (1973) used the catalog of energy curves of normal stars by Straizys \& Sviderskiene (1972), containing 18 O-M3 V stars, 3 G5-K0 IV stars, 14 A5-M6 III stars, 14 B0-M2 I stars. He used an absolute calibration based on the energy distribution curve of Vega according to the models by Mihalas (1966). The response curves for the Vilnius system have been taken from Straizys \& Zdanavicius (1970) and those of uvby - from Matsushima (1969).

The colour indices for the two systems has been normalized to zero for unreddened O-type stars. For all spectral type and luminosity classes, excluding only the Mtype giants and supergiants, he found the following linear relations:

$(u-b)-(U-Y)=0.024(b-y)_{0}-c E_{b-y}$

$(b-y)-(Y-V)=0.021(b-y)$, where $c$ varies from 0.005 to 0.016 with the spectral type.

Straizys et al. (1996a) showed that there are simple linear equations between the differences of the ultraviolet $U-u$, blue $Y-b$ and green $V-y$ magnitudes of the Vilnius and Strömgren systems as a function of $B-V$. As far as the interrelationship between the $X$ and $v$ magnitudes is concerned, it is non-linear over the temperature range and possibly affected by interstellar reddening.

\subsection{Empirical relationships}

Using the data collated in this paper, we investigate the relations between the colour indices $(U-Y) /(u-b)$, $(U-X) /(u-v),(X-Y) /(v-b)$ and $(Y-V) /(b-y)$. The first two of them are related to the Balmer discontinuity, while the last two are related to the metallicity and temperature, respectively. All relationships appear linear with small dispersion, although indeed different for the different spectral types. The coefficients of the linear fitting A and $\mathrm{B}$ for the different spectral types are given in Tables 1 to 4 . The standard errors of the coefficients and the parameters of the fitting (correlation coefficient $R$, standard error $S D$ and the number of points $N$ ) are also listed in these tables. Calculating these quantities, an error of $0.02 \mathrm{mag}$ was assumed in both Vlinius and Strömgren data. Figure 2 presents the coefficients $A$ and $B$ of the 
Table 1. The relation $U-Y=A+B(u-b)$ for different spectral types.

\begin{tabular}{ll}
\hline \hline Spectral type O & Spectral type B \\
$A=0.315 \pm 0.005$ & $A=0.331 \pm 0.007$ \\
$B=1.020 \pm 0.008$ & $B=1.020 \pm 0.008$ \\
$R=0.999$ & $R=0.998$ \\
$S D=0.020, N=31$ & $S D=0.035, N=141$ \\
Spectral type A & Spectral type F \\
$A=0.362 \pm 0.019$ & $A=0.353 \pm 0.01729$ \\
$B=0.999 \pm 0.013$ & $B=1.000 \pm 0.012$ \\
$R=0.981$ & $R=0.995$ \\
$S D=0.033, N=271$ & $S D=0.023, N=84$ \\
Spectral type G & Spectral type K \\
$A=0.396 \pm 0.019$ & $A=0.345 \pm 0.034$ \\
$B=0.984 \pm 0.011$ & $B=1.014 \pm 0.012$ \\
$R=0.996$ & $R=0.995$ \\
$S D=0.026, N=74$ & $S D=0.055, N=71$ \\
\hline
\end{tabular}

Table 2. The relation $(U-X)=A+B(u-v)$ for different spectral types.

\begin{tabular}{ll}
\hline \hline Spectral type O & Spectral type B \\
$A=0.218 \pm 0.003$ & $A=0.234 \pm 0.005$ \\
$B=0.962 \pm 0.010$ & $B=0.993 \pm 0.007$ \\
$R=0.998$ & $R=0.996$ \\
$S D=0.016, N=31$ & $S D=0.029, N=141$ \\
Spectral type A & Spectral type F \\
$A=0.207 \pm 0.017$ & $A=0.183 \pm 0.011$ \\
$B=1.000 \pm 0.014$ & $B=1.001 \pm 0.010$ \\
$R=0.976$ & $R=0.996$ \\
$S D=0.031, N=271$ & $S D=0.020, N=84$ \\
Spectral type G & Spectral type K \\
$A=0.206 \pm 0.014$ & $A=0.169 \pm 0.029$ \\
$B=0.938 \pm 0.013$ & $B=0.968 \pm 0.018$ \\
$R=0.993$ & $R=0.988$ \\
$S D=0.020, N=74$ & $S D=0.043, N=71$ \\
\hline
\end{tabular}

fitting as a function of the spectral type. Several stars involved in the comparison may be reddened, but the Vilnius and Strömgren indices should be affected in a similar way since they are alike. If the indices to be compared are on the same scale, meaning that the coefficient $B$ is $\approx 1$, any reddening will shift a point along the relation. If the scale differs, reddening will increase the scatter.

As far as the $(Y-V)$ and $(b-y)$ indices are concerned, there is an excellent agreement between the theoretically calculated relationship (Straizys 1973) and the empirical one, derived in this paper. The difference of $0.17 \mathrm{mag}$ in the zero point ( 0.15 for G-type stars, Table 4$)$ is due to the fact that we use Strömgren $(b-y)$ indices, which are not normalized to zero for an unreddened O-type star. This zero point difference is close to the average correction 0.15 mag calculated by Straizys (1977) for all spectral types.

The relation between $(X-Y)$ and $(v-b)$ appears to be more complex one because of the very complicated spectral region at $405-411 \mathrm{~nm}$, as also pointed out by Straizys (1973). It may indeed be seen in Fig. 2 that a gradient
Table 3. The relation $(X-Y)=A+B(v-b)$ for different spectral types.

\begin{tabular}{ll}
\hline \hline Spectral type O & Spectral type B \\
$A=0.089 \pm 0.003$ & $A=0.104 \pm 0.002$ \\
$B=1.098 \pm 0.010$ & $B=1.108 \pm 0.016$ \\
$R=1.000$ & $R=0.987$ \\
$S D=0.011, N=31$ & $S D=0.017, N=141$ \\
Spectral type A & Spectral type F \\
$A=0.135 \pm 0.003$ & $A=0.144 \pm 0.014$ \\
$B=1.074 \pm 0.013$ & $B=1.064 \pm 0.036$ \\
$R=0.981$ & $R=0.957$ \\
$S D=0.016, N=271$ & $S D=0.014, N=84$ \\
Spectral type G & Spectral type K \\
$A=0.185 \pm 0.013$ & $A=0.200 \pm 0.014$ \\
$B=1.062 \pm 0.019$ & $B=1.053 \pm 0.011$ \\
$R=0.989$ & $R=0.996$ \\
$S D=0.018, N=74$ & $S D=0.025, N=71$ \\
\hline
\end{tabular}

Table 4. The relation $(Y-V)=A+B(b-y)$ for different spectral types.

\begin{tabular}{ll}
\hline \hline Spectral type $\mathbf{O}$ & Spectral type B \\
$A=0.174 \pm 0.003$ & $A=0.169 \pm 0.001$ \\
$B=0.982 \pm 0.007$ & $B=0.975 \pm 0.010$ \\
$R=0.999$ & $R=0.993$ \\
$S D=0.012, N=31$ & $S D=0.017, N=141$ \\
Spectral type A & Spectral type F \\
$A=0.174 \pm 0.002$ & $A=0.172 \pm 0.005$ \\
$B=0.960 \pm 0.015$ & $B=0.974 \pm 0.022$ \\
$R=0.972$ & $R=0.982$ \\
$S D=0.019, N=271$ & $S D=0.012, N=84$ \\
Spectral type G & Spectral type K \\
$A=0.148 \pm 0.011$ & $A=0.161 \pm 0.011$ \\
$B=1.025 \pm 0.025$ & $B=0.985 \pm 0.016$ \\
$R=0.979$ & $R=0.992$ \\
$S D=0.015, N=74$ & $S D=0.019, N=71$ \\
\hline
\end{tabular}

in the coefficient $A$ exists for the $(X-Y) /(v-b)$ relation. It may be caused by the decreasing equivalent width (EQW hereafter) of $\mathrm{H} \delta$ line toward the cooler stars, which affects $X$ and $v$ pass-bands in a relatively different way. The pass-bands $X$ is less affected because it is shifted and slightly wider. Also, it may be a temperature effect that influences the $X$ and $v$ bands in a different way since the $\mathrm{CaII} \mathrm{H}$ and $\mathrm{K}$ lines become more pronounced for cooler stars. It could also be an effect on $Y$ and $b$ pass-bands, since the same $A$-gradient is present in the $(U-Y) /(u-b)$ comparison.

Apparently the effects of metallicity and luminosity may affect the Vilnius and Strömgren indices in a different way, despite their similarities. It should be mentioned however, that these effects cannot be fully separated from each other. To investigate in more details the effects of luminosity and metallicity that emerge in the comparison, the differences between the corresponding colour indices are plotted as a function of the Strömgren index in the first four panels in Fig. 3. These plots in general repeat the trends seen in Fig. 2. The last four panels in Fig. 3 

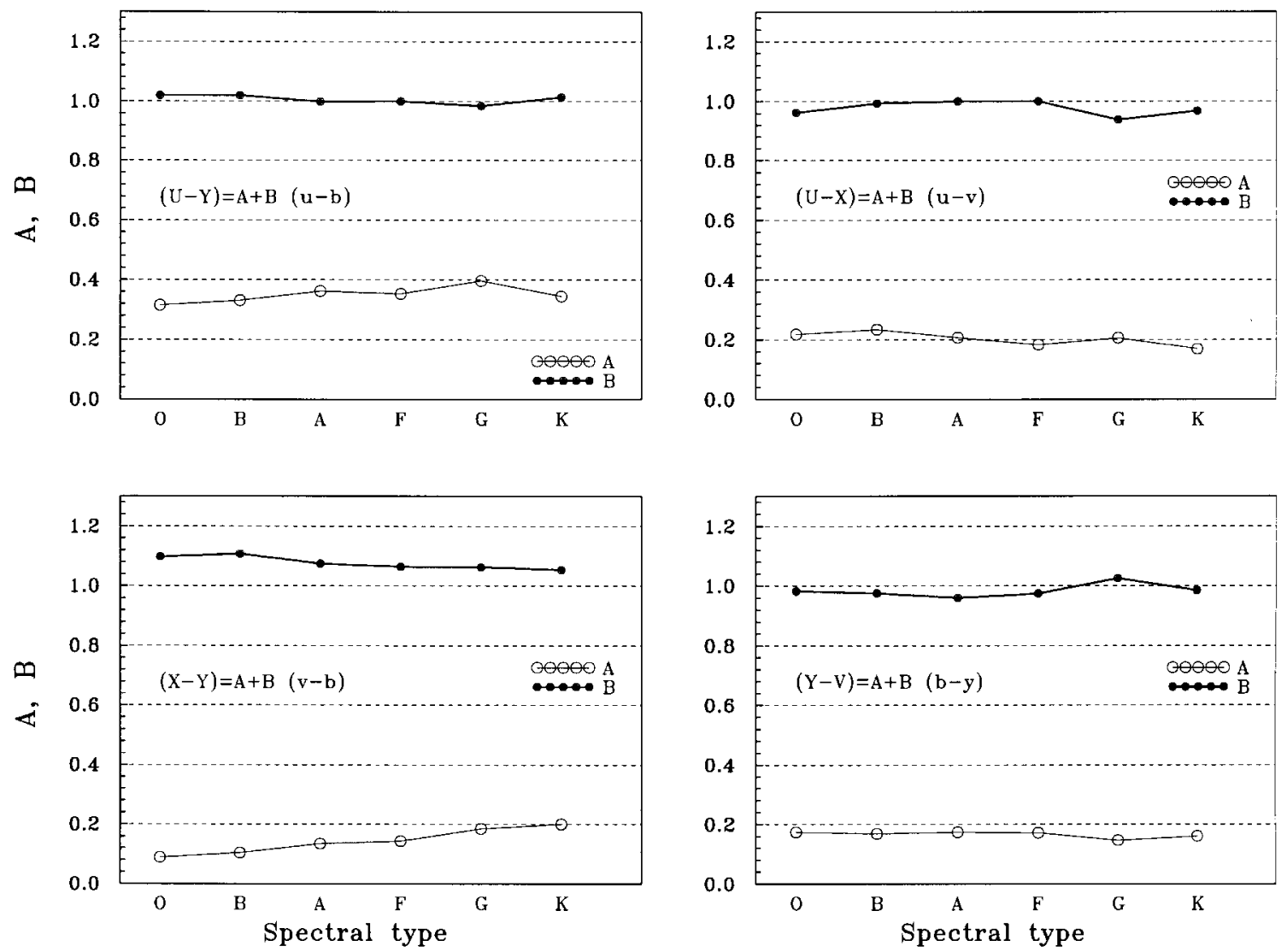

Fig. 2. The coefficients A and B from Tables 1 to 4 plotted as a function of the spectral type. Since the error-bars are small and of about the size of the symbols used, they are not shown.

present the comparison of $c_{1}=(u-v)-(v-b)$ and $m_{1}=(v-b)-(b-y)$ to the corresponding quantities in the Vilnius system. To facilitate the visualization regarding the different sub-samples, different $y$-scale is used in panels a-d. The plots show complex behavior when $v$ and $X$ magnitudes are involved (panels $\mathrm{b}$ and $\mathrm{c}$ ). It may be seen that the discrepancy between $(v-b)$ and $(X-Y)$ is about 10 times larger than for $(b-y)$ and $Y-V$. Interestingly, $(u-v)$ and $(U-X)$ show a good agreement for O-A spectral sequence, but different trend for $\mathrm{G}$ and K-types. The discrepancies between $(b-y)$ and $(Y-V)$ and $(u-b)$ and $(U-Y)$ are mostly due to blanketing, which influences differently these pass-bands, being stronger toward the short wavelengths. The $[\mathrm{Fe} / \mathrm{H}]$ influence and the luminosity effect may possibly be visible in a $(X-Y)-(Y-V)$ $\left(m_{1}\right.$ (Vil) hereafter $)$ vs. $m_{1}=(v-b)-(b-y)$ comparison and in a $(U-X)-(X-Y)\left(c_{1}\right.$ (Vil) hereafter $)$ vs. $c_{1}=(u-v)-(v-b)$ comparison (Figs. 3e, g). Since the split in Fig. 3g occurs just at the Balmer maximum one would guess that the different influence of $\mathrm{H} \delta$ on $X$ and $v$ was the cause. On the other hand the metal abundance may influence $X$ more than $v$ and the metals influence the cool but not the hot stars $(X$ mag $(\mathrm{cool})>X$ mag (hot)). For the cool stars there is again a shift from $\mathrm{F}$ to $\mathrm{G}$ type stars and from A to $\mathrm{F}$ spectral type. The diagrams in panels $\mathrm{f}$ and $\mathrm{h}$ present $m_{1}$ as a function of the difference $\Delta m_{1}=m_{1}(\mathrm{Vil})-m_{1}$, and respectively about $c_{1}$.
The A-K stars of Fig. 3f show a distribution like the similar range of stars in a $m_{1}$ vs. $(b-y)$ diagram. The $\mathrm{O}$ and B stars are shifted toward smaller $m_{1}$ values and large values of $m_{1}(\mathrm{Vil})-m_{1}$ due to reddening. The difference $\Delta m_{1}$ behaves apparently like the temperature index $(b-y)$, so some $T_{\text {eff }}$ dependence is left in either $m_{1}$ (Vil) and $m_{1}$.

In Fig. 3h we see a distribution of $c_{1}$ vs. $\Delta c_{1}=$ $c_{1}$ (Vil) $-c_{1}$ similar to the standard $c_{1}$ vs. $m_{1}$ relation. Again we notice that the spread in $\Delta c_{1}$ for the O stars toward small values is like for $m_{1}$ influenced by reddening. So the difference of $m_{1}$ and $c_{1}$ in the two systems seems to have some metallicity and/or temperature dependence, likely due to the $X$ and $v$ magnitudes. To further analyse the effects that cause different photometric behavior of the $X$ and $v$ magnitudes, we plotted $(v-y)-(X-V)$ vs. $(b-y)$ and $(v-y)-(X-V)$ vs. $\beta$ diagrams (Fig. 4$)$. These diagrams are helpful to make a distinction between a $\mathrm{H} \delta$ effect and a metal abundance effect. The $(v-y)-(X-V)$ vs. $(b-y)$ diagram shows a trend with the spectral type, apart from the upper branch, due to reddening, and a few deviant $\mathrm{K}$ stars. This trend may be owing to an increase of metallicity, thus to an increase of the CaII lines strength toward the cooler stars. The $(v-y)-(X-V)$ vs. $\beta$ diagram demonstrates a very strong dependence on temperature for the late $\mathrm{F}$ subtypes and $\mathrm{G}$ and $\mathrm{K}$ types, where the $\mathrm{H} \beta$ line is week, or not present. The earlier spectral types show a very small change of the $(v-y)-(X-V)$ 
N. Kaltcheva and J. Knude: Transformations between Vilnius and Strömgren
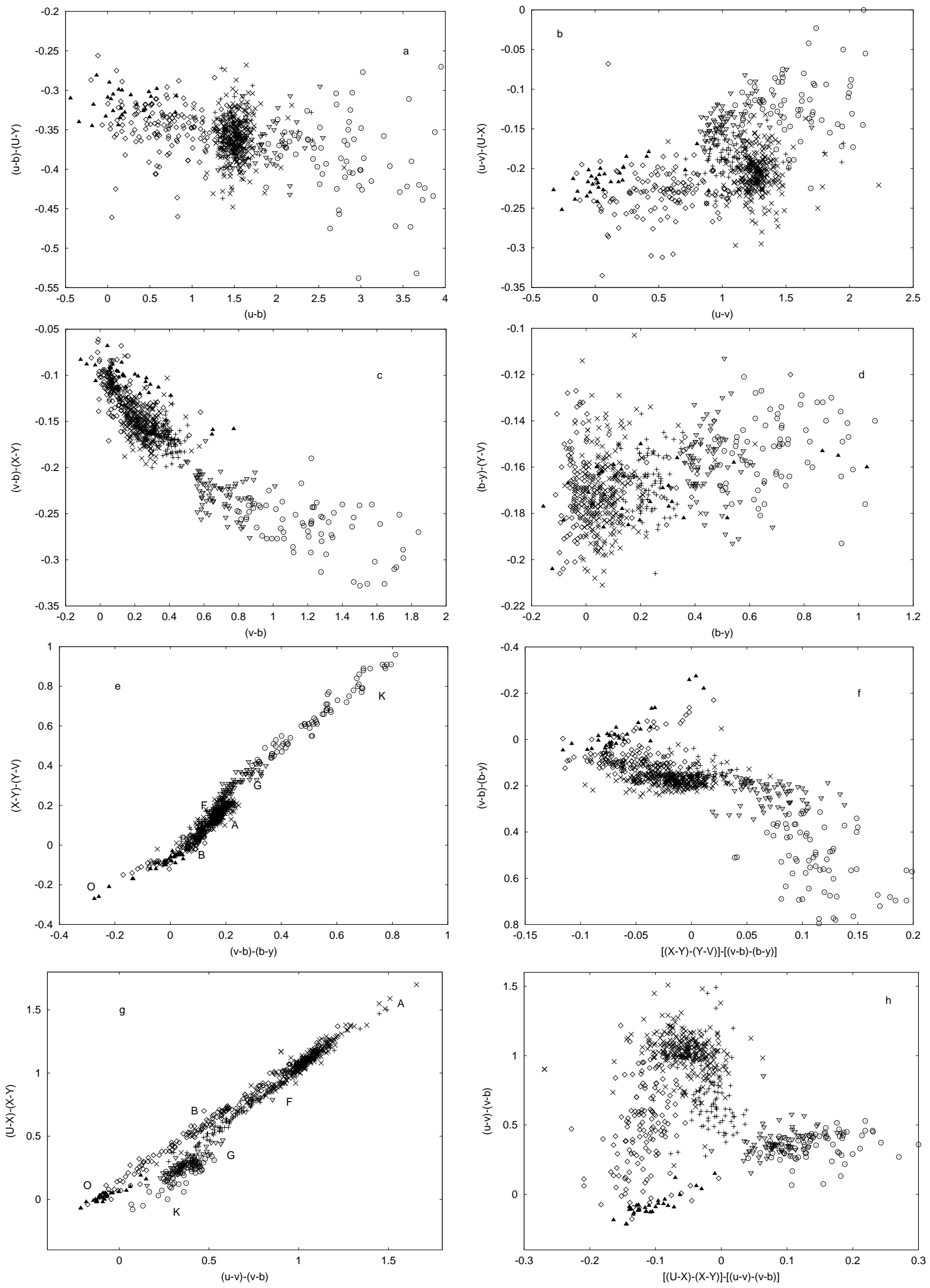

Fig. 3. Dependencies between corresponding Vilnius and Strömgren indices and colour differences. Different symbols are used for all sub-samples as follows: filled triangles: O-type, open diamonds: B-type, crosses: A-type, plus symbols: F-type, open triangles: G-type, open circles: K-type. 

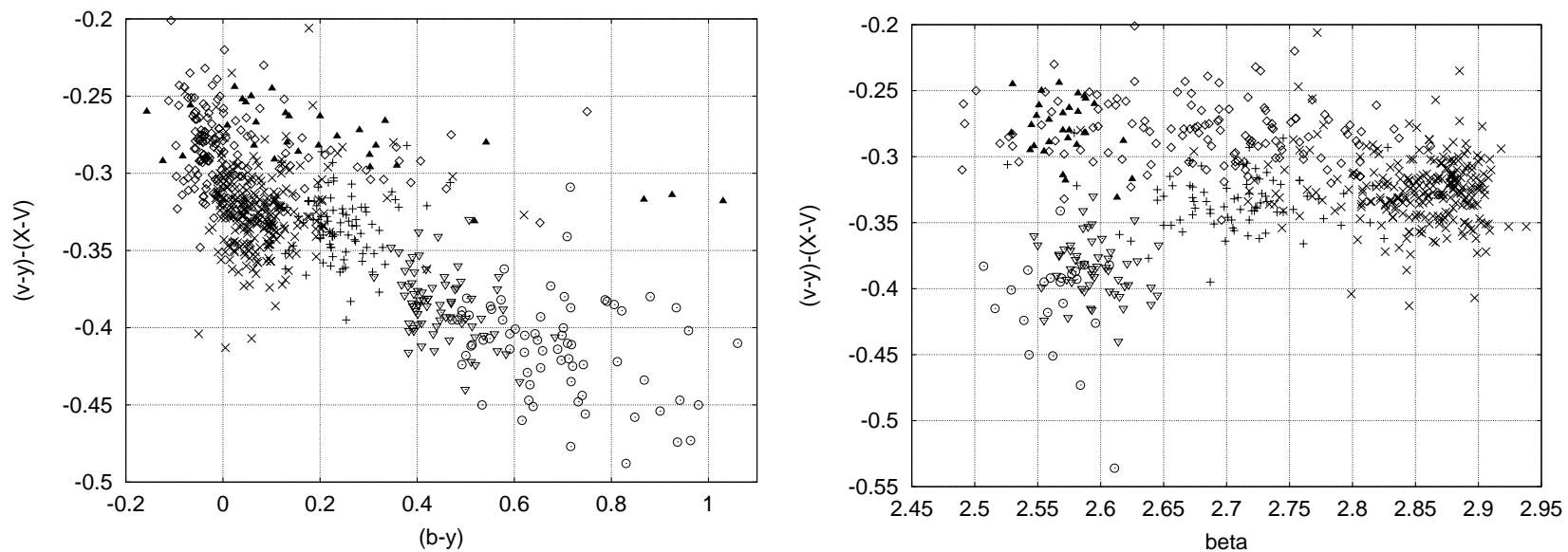

Fig. 4. The $(v-y)-(X-V)$ vs. $b-y$ and $(v-y)-(X-V)$ vs. $\beta$ diagrams for all sub-samples. The symbols are as in Fig. 3 .

Table 5. The sample used for Figs. 5b-f. The stars are grouped by spectral type and luminosity class. HD number is given in the first column and the Strömgren and Vilnius photometric data are listed in Cols. 2-12. Columns 13 and 14 present the star name and MK classification. Columns 15-17 contain data for $\log g,[\mathrm{Fe} / \mathrm{H}]$ and EQW (in units $0.1 \mathrm{~nm}$ ) of Ca II T adopted from Mallik (1997).

\begin{tabular}{|c|c|c|c|c|c|c|c|c|c|c|c|c|c|c|c|c|}
\hline HD & $(b-y)$ & $m_{1}$ & $c_{1}$ & $\beta$ & $V$ & $U-P$ & $P-X$ & $X-Y$ & $Y-Z$ & $Z-V$ & $V-S$ & Name & MK & $\log g$ & {$[\mathrm{Fe} / \mathrm{H}]$} & $\begin{array}{l}\text { EQW } \\
\text { Ca T }\end{array}$ \\
\hline & mag & mag & mag & mag & $\mathrm{mag}$ & $\mathrm{mag}$ & mag & $\mathrm{mag}$ & mag & $\mathrm{mag}$ & mag & & & & Sun & $0.1 \mathrm{~nm}$ \\
\hline \multicolumn{17}{|l|}{ K II-Ib } \\
\hline 200905 & 1.024 & 0.696 & 0.218 & 2.611 & 3.72 & 0.81 & 1.30 & 2.08 & 0.67 & 0.53 & 1.07 & $\xi$ Cyg & $\mathrm{K} 4 \mathrm{Ib}$ & 1.60 & -0.01 & 11.97 \\
\hline 206778 & 0.963 & 0.681 & 0.299 & 2.584 & 2.39 & 0.81 & 1.28 & 1.97 & 0.66 & 0.45 & 0.96 & $\epsilon \mathrm{Peg}$ & $\mathrm{K} 2 \mathrm{Ib}$ & 1.00 & -0.03 & 11.98 \\
\hline 180809 & 0.787 & 0.564 & 0.332 & 2.587 & 4.36 & 0.69 & 1.13 & 1.60 & 0.55 & 0.37 & 0.84 & $\theta \mathrm{Lyr}$ & KOII & 1.72 & -0.20 & 10.64 \\
\hline 186791 & 0.936 & 0.764 & 0.290 & & 2.72 & 0.76 & 1.34 & 2.01 & 0.63 & 0.47 & 0.97 & $\gamma \mathrm{Aql}$ & K3II & 2.40 & 0.00 & 9.69 \\
\hline \multicolumn{17}{|c|}{ K V-IIIb } \\
\hline 23249 & 0.558 & 0.372 & 0.393 & & 3.54 & & & 1.20 & 0.41 & 0.30 & 0.70 & $\delta$ Eri & KOIV & 3.95 & 0.05 & 7.08 \\
\hline 29139 & 0.955 & 0.814 & 0.373 & & 0.85 & 0.79 & 1.40 & 2.07 & 0.56 & 0.54 & 1.07 & $\alpha \mathrm{Tau}$ & K5III & 1.50 & 0.00 & 9.30 \\
\hline 37160 & 0.591 & 0.314 & 0.419 & & 4.09 & 0.58 & 0.86 & 1.17 & 0.44 & 0.30 & 0.75 & $\phi^{2}$ Ori & KOIII & 2.40 & -0.70 & 6.57 \\
\hline 96833 & 0.703 & 0.524 & 0.396 & & 3.01 & 0.62 & 1.11 & 1.47 & 0.48 & 0.36 & 0.80 & $\psi \mathrm{UMa}$ & K1III & 2.78 & -0.07 & 8.74 \\
\hline 107328 & 0.718 & 0.483 & 0.516 & 2.570 & 4.96 & 0.66 & 1.15 & 1.47 & 0.49 & 0.37 & 0.85 & $16 \mathrm{Vir}$ & KOIIIb & 2.30 & -0.20 & 8.23 \\
\hline 124897 & 0.753 & 0.531 & 0.488 & & -0.04 & 0.72 & 1.17 & 1.57 & 0.51 & 0.40 & 0.87 & $\alpha$ Boo & K1IIIb & 1.70 & -0.60 & 7.64 \\
\hline 137759 & 0.712 & 0.566 & 0.433 & & 3.29 & 0.64 & 1.20 & 1.55 & 0.48 & 0.38 & 0.80 & $\iota$ Dra & K2III & 2.60 & 0.30 & 8.75 \\
\hline 140573 & 0.715 & 0.571 & 0.451 & & 2.65 & 0.59 & 1.17 & 1.54 & 0.47 & 0.30 & 0.78 & $\alpha$ Ser & K2III & 2.90 & 0.37 & 7.71 \\
\hline 164058 & 0.941 & 0.811 & 0.373 & & 2.23 & 0.80 & 1.38 & 2.05 & 0.56 & 0.53 & 1.02 & $\gamma$ Dra & K5III & 1.55 & -0.23 & 10.02 \\
\hline 198149 & 0.553 & 0.366 & 0.375 & & 3.43 & 0.53 & 0.88 & 1.16 & 0.42 & 0.28 & 0.71 & $\eta$ Cер & KOIV & 3.00 & -0.50 & 6.47 \\
\hline \multicolumn{17}{|c|}{ G IIIa-Ib } \\
\hline 48329 & 0.860 & 0.678 & 0.273 & 2.615 & 2.98 & 0.73 & 1.16 & 1.79 & 0.63 & 0.39 & 0.86 & $\epsilon \mathrm{Gem}$ & G8Ib & 0.80 & -0.05 & 11.35 \\
\hline 218356 & 0.835 & 0.535 & 0.164 & 2.566 & 4.76 & 0.64 & 0.98 & 1.59 & 0.57 & 0.41 & 0.92 & $56 \mathrm{Peg}$ & G8Ib & 1.40 & -0.20 & 7.78 \\
\hline 84441 & 0.507 & 0.276 & 0.452 & 2.594 & 2.98 & 0.57 & 0.77 & 1.00 & 0.42 & 0.20 & 0.64 & $\epsilon$ Leo & G1II & 2.40 & -0.13 & 8.17 \\
\hline 92125 & 0.512 & 0.294 & 0.478 & 2.595 & 4.71 & 0.64 & 0.77 & 1.09 & 0.42 & 0.23 & 0.66 & $37 \mathrm{LMi}$ & G2II & 1.87 & 1000 & 10.29 \\
\hline 148897 & 0.817 & 0.382 & 0.540 & 2.547 & 5.25 & 0.79 & 1.05 & 1.54 & 0.61 & 0.36 & 0.93 & & G8II & 1.50 & -0.62 & 7.95 \\
\hline \multirow{2}{*}{\multicolumn{17}{|c|}{ G V-III }} \\
\hline & & & & & & & & & & & & & & & & \\
\hline 10700 & 0.443 & 0.253 & 0.244 & 2.586 & 3.50 & 0.43 & 0.68 & 0.90 & 0.33 & 0.25 & 0.61 & $\tau$ Cet & G8V & 4.50 & -0.58 & 5.08 \\
\hline 34411 & 0.390 & 0.205 & 0.364 & 2.600 & 4.71 & 0.46 & 0.65 & 0.83 & 0.34 & 0.22 & 0.56 & $\lambda$ Aur & G2IV-V & 4.10 & 0.30 & 7.34 \\
\hline 103095 & 0.484 & 0.222 & 0.155 & 2.547 & 6.42 & 0.44 & 0.60 & 0.93 & 0.36 & 0.26 & 0.65 & & G9VI & 4.50 & -1.40 & 5.20 \\
\hline 114710 & 0.368 & 0.192 & 0.338 & 2.608 & 4.26 & 0.46 & 0.59 & 0.78 & 0.33 & 0.19 & 0.55 & $\beta$ Com & G0V & 4.40 & 0.02 & 5.21 \\
\hline 121370 & 0.374 & 0.203 & 0.488 & 2.629 & 2.68 & 0.50 & 0.67 & 0.79 & 0.32 & 0.22 & 0.50 & $\eta$ Boo & G0IV & 3.80 & 0.16 & 4.90 \\
\hline 135722 & 0.589 & 0.346 & 0.407 & & 3.47 & 0.58 & 0.89 & 1.19 & 0.46 & 0.30 & 0.71 & $\delta$ Boo & G8III & 2.70 & -0.50 & 6.76 \\
\hline 161797 & 0.468 & 0.271 & 0.407 & 2.614 & 3.42 & 0.50 & 0.80 & 0.98 & 0.37 & 0.25 & 0.62 & $\mu$ Her & G5IV & 4.10 & 0.32 & 6.86 \\
\hline 182572 & 0.470 & 0.287 & 0.408 & 2.597 & 5.16 & 0.49 & 0.84 & 0.98 & 0.36 & 0.27 & 0.60 & $31 \mathrm{Aql}$ & G8IV & 4.00 & 0.21 & 7.17 \\
\hline 188512 & 0.520 & 0.306 & 0.343 & 2.555 & 3.71 & 0.50 & 0.80 & 1.09 & 0.40 & 0.28 & 0.66 & $\beta \mathrm{Aql}$ & G8IV & 3.79 & -0.15 & 6.73 \\
\hline 222107 & 0.625 & 0.314 & 0.408 & 2.554 & 3.82 & 0.60 & 0.89 & 1.22 & 0.43 & 0.36 & 0.75 & $\lambda$ And & G8III-IV & 2.30 & -0.78 & 5.15 \\
\hline \multicolumn{17}{|l|}{ F V } \\
\hline 9826 & 0.346 & 0.176 & 0.415 & 2.629 & 4.09 & 0.48 & 0.62 & 0.71 & 0.31 & 0.22 & 0.48 & $v$ And & $\mathrm{F} 8 \mathrm{~V}$ & 3.91 & -0.23 & 5.27 \\
\hline 102870 & 0.354 & 0.187 & 0.416 & 2.628 & 3.61 & 0.46 & 0.65 & 0.73 & 0.33 & 0.17 & 0.49 & $\beta$ Vir & F9V & 4.10 & 0.18 & 4.72 \\
\hline
\end{tabular}

with $\beta$. The A-type stars present no dependence until A0, and then the upper points display a shallow variation through the $\mathrm{B}$ and $\mathrm{O}$ star range. The reason of increasing $(v-y)-(X-V)$ values toward the early B and O stars may be due to the fact that they are more reddened and more luminous in general in comparison to the A-type stars. Since $(v-y)-(X-V)$ behaves in a similar way as $m_{1}$, we expect $(v-y)-(X-V)$ for the earlier types to be smaller, due to their intrinsically smaller values and their possible large colour excess. The $m_{1}$ range for O-A0 stars is very small in the Strömgren system, of about 0.05-0.15 mag, smaller for the earliest types. On the average earlier stellar types will be more reddened than the later types in this spectral range because they are more luminous and our sample is approximately apparent magnitude limited. The separation of the $(v-y)-(X-V)$ values for small values of $\beta$ may be due to an increase of the strength of Ca II toward F-G and $\mathrm{K}$ types. An increased EQW of Ca II will cause a decrease of the $(v-y)-(X-V)$ difference. Thus, at this point we suggest that the $(v-X)$ difference is predominantly caused by the Ca II H and K spectral feature. 

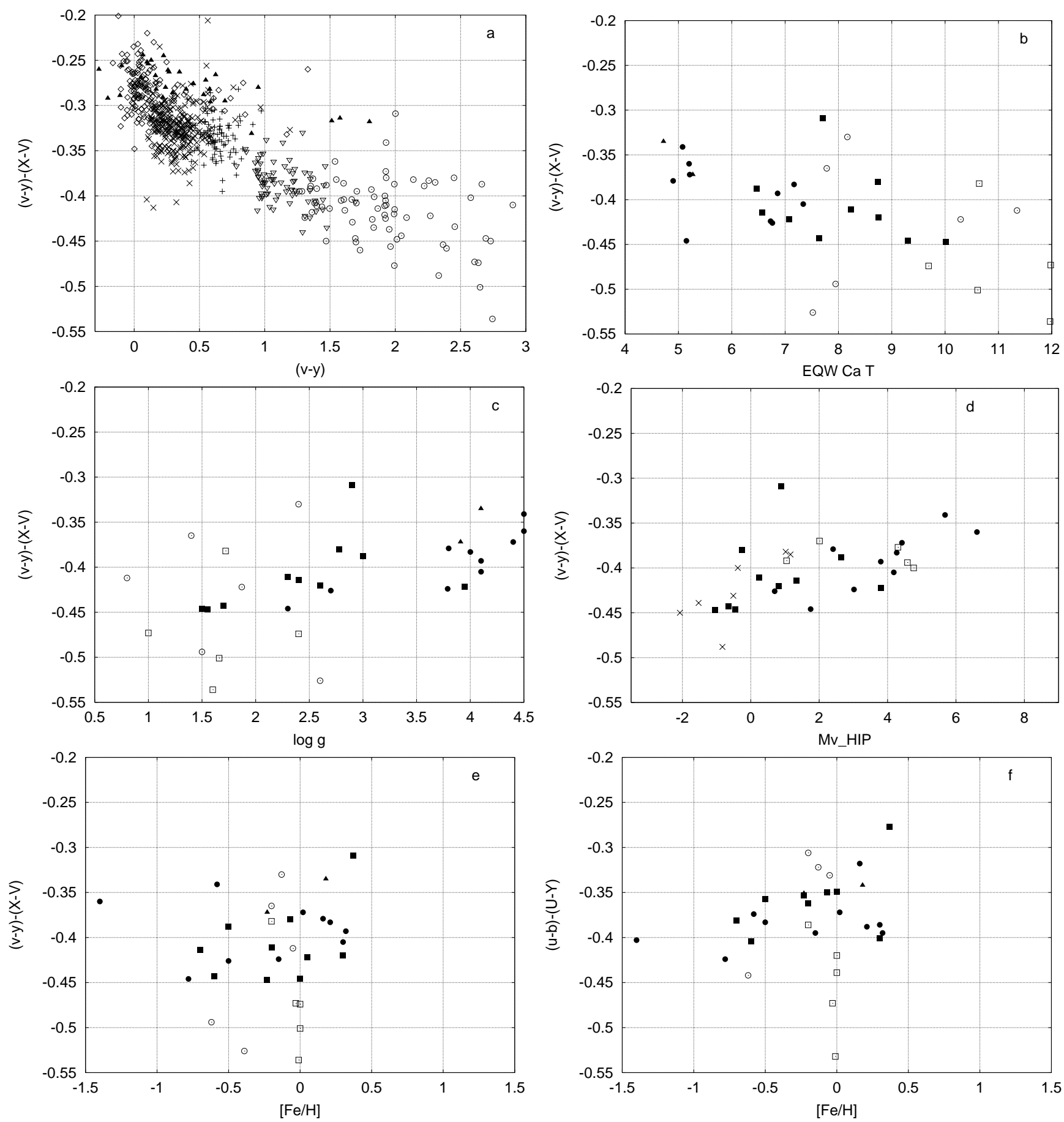

Fig. 5. a) The difference $(v-y)-(X-V)$ as a function of $(v-y)$ (all sub-samples are plotted and the symbols are the same as in Fig. 3). b), c), d) the difference $(v-y)-(X-V)$ as a function of EQW Ca II T, $\log g$ and $M_{\mathrm{V}} \mathrm{HIP}$ for G and K type stars with EQW Ca II T data available. e), f) The difference $(v-y)-(X-V)$ and $(u-b)-(U-Y)$ as a function of $[\mathrm{Fe} / \mathrm{H}]$ for the same sample. The symbols in the plots $\mathbf{b}-\mathbf{f})$ are as follows: open symbols - stars more luminous than LC III, filled symbols - LC V, IV, III, circles - G-type, squares - K-type. The two late F-type dwarfs available are plotted with filled triangles.

To check this hypothesis we searched the literature for measurement of the EWQ of Ca II stellar lines. Several authors have presented measurements of the EQW of CaII infrared triplet (CaII T hereafter). If we assume that a correlation exists between the strength of the Ca II T lines in the infrared and in the visual region of the spectra, we may use the existing Ca II T data-bases to investigate the influence of CaII on $(v-X)$. This assumption is rather justified since the CaII $\mathrm{H}$ and $\mathrm{K}$ lines have the same upper level as the Ca II T. The largest homogeneous sample of stars with both Vilnius, Strömgren and Ca II T data can be collated from the data-base of Mallik (1997). This 

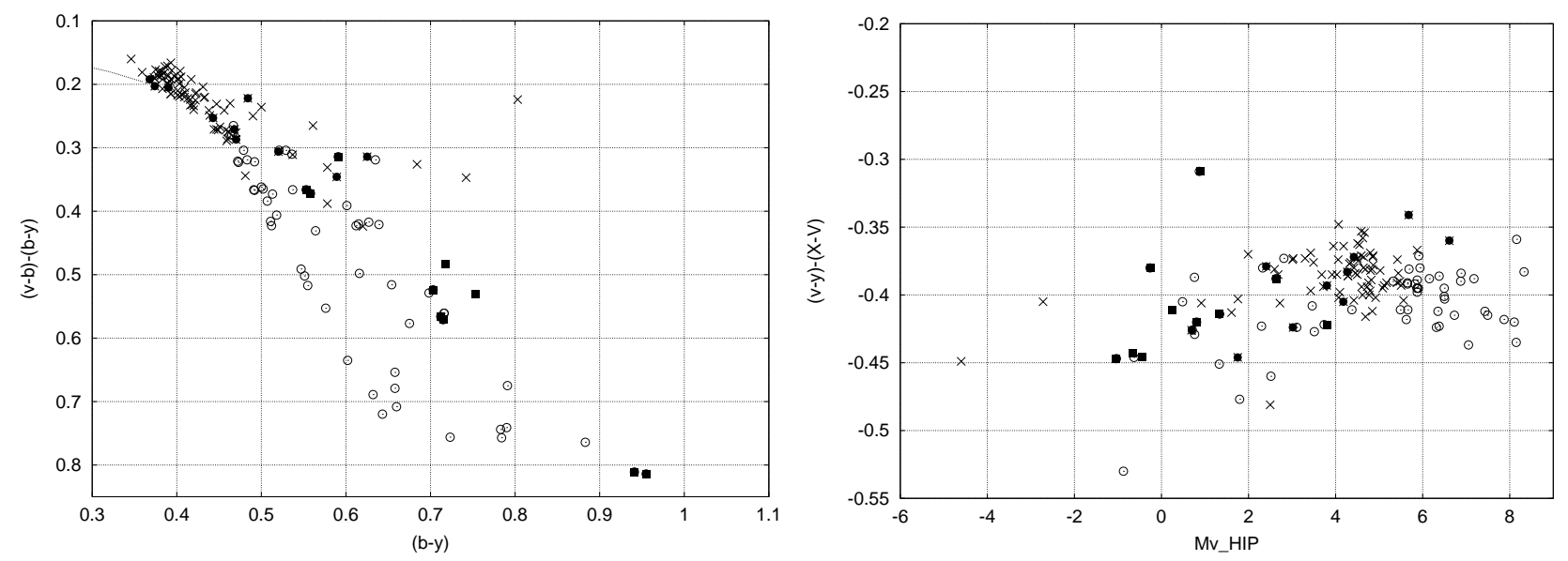

Fig. 6. $\mathrm{G}$ and $\mathrm{K}$ type stars within 50 pc from the Sun with Vilnius and Strömgren photometry and Hipparcos parallaxes available: the $m_{1}$ vs. $(b-y)$ diagram (left) and the difference $(v-y)-(X-V)$ as a function of $M_{V}$ HIP (right). Crosses mark the G-type stars (all luminosity classes), open circles - K-type stars (all luminosity classes). The sample of stars of moderate luminosity used in Fig. 5 is shown with filled symbols (same as in Fig. 5).

sample is given in Table 5, where the stars are grouped according to the spectral and luminosity class. Please, note, that the sample in Table 5 is not restricted according to the $Q_{\mathrm{X} Y V}$ parameter. Thus, it contains several stars that are not included in the sub-samples used in the general comparison of the photometric indices. For example, the two FV stars in Table 5 are not included in the Ftype sub-sample, because their $Q_{\mathrm{X} Y V}$ values are larger than $Q_{\mathrm{XY} V}<0.3 \mathrm{mag}$ restriction for F-type stars in the Vilnius system. However, as mentioned before, such stars do not show any peculiar photometric behavior and are classified via spectra, and we include them in the following discussion to assure the largest sample possible. The first column of Table 5 presents the star identification and the Strömgren and the Vilnius photometry follow. The name of the stars and the MK classifications are given in Cols. 13 and 14. The last three columns contains the data for $\log g$, metallicity and the EQW of CaII T which are adopted from Mallik (1997).

In order to investigate the Ca II influence on the $(v-X)$ index, $(v-y)-(X-V)$ is plotted vs. $(v-y)$ for all subsamples in Fig. 5a. Figures $5 \mathrm{~b}, \mathrm{c}$, d show the dependence of $(v-y)-(X-V)$ of Ca II T, $\log g$ and the absolute magnitude $M_{\mathrm{V}}$ derived from the Hipparcos (ESA 1997) parallaxes for the stars listed in Table 5 . Figures $5 \mathrm{e}, \mathrm{f}$ present the dependence of $(v-y)-(X-V)$ and $(u-b)-(U-Y)$ of the metallicity index $[\mathrm{Fe} / \mathrm{H}]$. Please, note that the sample in Table 5 is restricted only to spectral type $\mathrm{G}$ and $\mathrm{K}$, where CaII $\mathrm{T}$ data are available, and so are the plots Figs. 5b-f. Different MK types are marked with different symbols to separate stars of moderate luminosity (LC V, IV, III, IIIb) and stars of higher luminosity (IIIa, II, Ib), as explained in the figure's caption. The $(v-y)-(X-V)$ vs. $(v-y)$ plot (Fig. 5a) may be compared to Fig. 3 from the comparative analysis of the observed colour indices in the Vilnius system and theoretical spectral energy distri- butions for $[\mathrm{Fe} / \mathrm{H}]=0$ presented by Valiauga et al. (1996). Note, that $(v-y)$ should be corrected with 0.23 to match the zero point of the Vilnius indices. The range of the $(v-y)-(X-V)$ seems about the same as in Fig. 3 of Valiauga et al. (1996), but the slope is significantly larger. It should be mentioned that Valiauga et al. (1996) degrade both the theoretical and the observational spectral scans to $5 \mathrm{~nm}$, so any effects due to CaII lines in the $X$ filter would be smoothed out in their comparison. Our Fig. 5a may be also compared to Fig. 4 (second panel) of Straizys et al. (1996), which shows a similar trend. Thus, (since the Strömgren $(v-y)$ should not be significantly influenced by CaII lines), Fig. 5a should present mainly the change of the $X$ magnitude with the strength of the CaII lines over the spectral sequence.

As pointed out by Mallik (1994, 1997), the CaII T is bi-parametric with a strong dependence on luminosity and a milder dependence of metallicity. In Figs. 5b, c the dependence of the $(v-y)-(X-V)$ difference on the CaII $\mathrm{T}$, which is a log $g$ indicator, almost independent on $[\mathrm{Fe} / \mathrm{H}]$ (Fig. 5e) is clearly seen. This indicates that the $(v-X)$ index may be used as a luminosity indicator with very modest scatter of approximately 0.2 in $\log g$, that would be very useful for late type stars of moderate luminosity. Since $v$ and $X$ have almost identical central wavelength, 411 and $405 \mathrm{~nm}$ respectively, the index $(v-X)$ is little affected by reddening, i.e. it is a reddening free $\log g$ indicator. This may be of importance for the late type dwarfs where reddening correction is difficult: if e.g. GAIA mission (ESA 2000) provides the distance, $(v-X)$ gives $\log g$, then $A_{\mathrm{V}}$ may be calculated. The same trend as in Fig. $5 \mathrm{c}$ is present in the comparison to the $M_{\mathrm{V}}$ values inferred from the Hipparcos parallaxes. The plots leave no doubt that the $X$ magnitude is luminosity dependent, owing to the Ca II lines at $394 \mathrm{~nm}$. Since some of the stars 

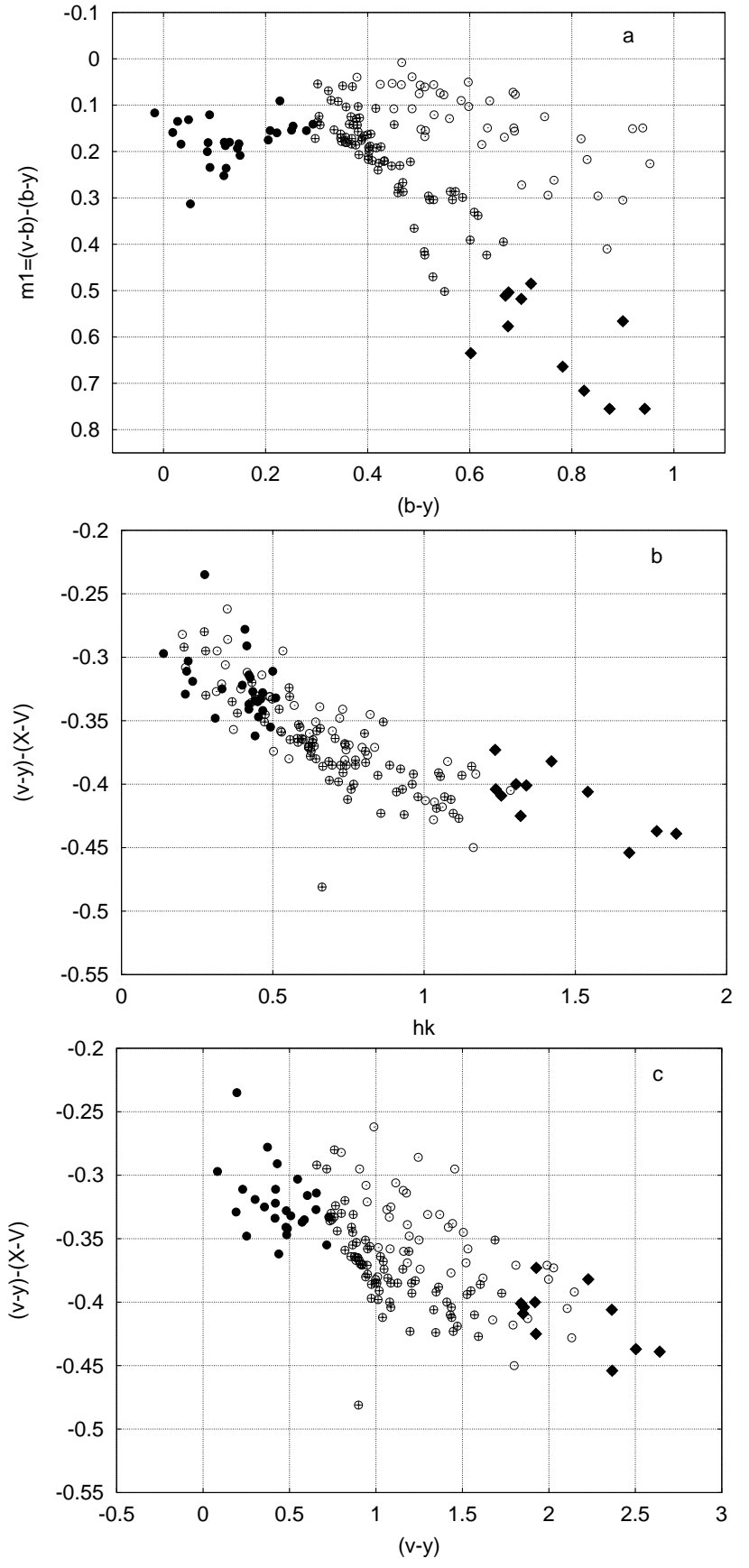

Fig. 7. a) filled symbols: F-type stars; open symbols: G and K-type of moderate/high luminosity; open symbols with plus sign: $\mathrm{G}$ and K-type of low/moderate luminosity; filled diamonds: late K-type dwarfs. b) $(v-y)-(X-V)$ differences vs. $h k$. c) $(v-y)-(X-V)$ differences vs. $(v-y)$ indices. Different symbols are used for different spectral and luminosity types, as distinguished based on the the $m_{1}$ vs. $(b-y)$ diagram.

in Table 5 have $\mathrm{H} \beta$ photometry, we studied $(v-y)-(X-$ $V)$ vs. $\beta$ relation as well, which, as expected, showed no dependence for $\mathrm{G}-\mathrm{K}$ spectral range (see Fig. 4b).

Figure 5f presents the differences $(u-b)-(U-Y)$ plotted vs. $[\mathrm{Fe} / \mathrm{H}]$. Contrary to the $(v-y)-(X-V)$ vs. $[\mathrm{Fe} / \mathrm{H}]$ plot in Fig. 5e, the $(u-U)$ index show smaller scatter and may be used for an estimation of the $[\mathrm{Fe} / \mathrm{H}]$ index with an accuracy of about 0.4 dex. As shown by Jønch-Sørensen (1995) an accuracy of this order could be used to discuss the chemical gradient in the Galaxy based on F and early G-type stars.

We realize that the number of stars with CaII T measurement and data available in both photometric systems is small and the sample in Table 5 is not sufficient for more global conclusions. The sample is biased regarding the MK types as well - it does not contain any $\mathrm{K}$ dwarfs and any $\mathrm{G}$ and $\mathrm{K}$ supergiants. As it may be seen in Fig. 5d, the Hipparcos $M_{\mathrm{V}}$ may be also used to evaluate the influence of $\log g$ on the $X$ pass-band for a larger sample, more uniform over the MK types. Figure 6 presents the $m_{1}$ vs. $(b-y)$ diagram and the difference $(v-y)-(X-V)$ as a function of $M_{\mathrm{V}} \mathrm{HIP}$ for a larger sample of $\mathrm{G}$ and $\mathrm{K}$ type stars. Only $\mathrm{G}$ and $\mathrm{K}$ type stars within $50 \mathrm{pc}$ from the Sun with Vilnius and Strömgren photometry and Hipparcos parallaxes available are included, in order to avoid reddened stars. From Fig. 6, it may be concluded, that the trend seen in Fig. 5 is actually the influence of the luminosity change from $G$ dwarfs to $K$ sub-giants and giants on the $X$ pass-band. Possibly, the effect is weaker in K-type dwarfs, due to less strong Ca II lines. Although restricted of lack of data, a complex behavior of the $(v-y)-(X-V)$ difference over the $\mathrm{G}-\mathrm{K}$ spectral sequence is evident, apparently owing to Ca II lines in the $X$ pass-band.

Anthony-Twarog et al. (1991) introduced a fifth filter $\mathrm{Ca}(f w h m=90 \mathrm{~A})$ centered on $\mathrm{CaII} \mathrm{H}$ and $\mathrm{K}$ lines to be used with the standard uvby system. A (metallicity) index, $h k$, is defined by replacing $v$ in $m_{1}$ by Ca. It is designed primarily for applications to metal-poor dwarfs and red giants, where the uvby metallicity index $m_{1}$ loses some sensitivity. $h k$ is more sensitive than $m_{1}$ over the regions of interest by about a factor of 3 . The effects of interstellar extinction on the $h k$ index are demonstrated to be modest and relatively insensitive to spectral type. Twarog \& Anthony-Twarog (1995) have published $h k$ indices for almost 2000 stars. Figure 7 presents a comparison of $(v-X)$ to the $h k$ index for the stars in common. The stars from this comparison sub-sample cover a spectral range from mid $\mathrm{F}$ to mid $\mathrm{K}$ types (Fig. 7a). As it may be seen from Fig. $7 \mathrm{~b}$, the dependence of $(v-X)$ vs. $h k$ does not show a separation on luminosity or spectral type until spectral type K. The separation of the K-type dwarfs in Fig. $7 \mathrm{~b}$ is possibly due to the decrease of the strength of the $\mathrm{Ca}$ II $\mathrm{H}$ and $\mathrm{K}$ lines toward this type, so the temperature effect which influences differently $(v-X)$ and $h k$ is more noticeable. Contrary, the $(v-X)$ vs. $(v-y)$ dependence (Fig. 7c) shows (as Fig. 5a) a luminosity class and spectral type separation, which is due to the $X$ magnitude. This points out again that $(v-X)$ index reflects the strength of the Ca II H and $\mathrm{K}$ stellar lines in a similar way as the $h k$ index, i.e. the $(v-X)$ index may be indeed used as a measure of the strength of $\mathrm{CaII} \mathrm{H}$ and $\mathrm{K}$ lines with a remarkably small scatter. 

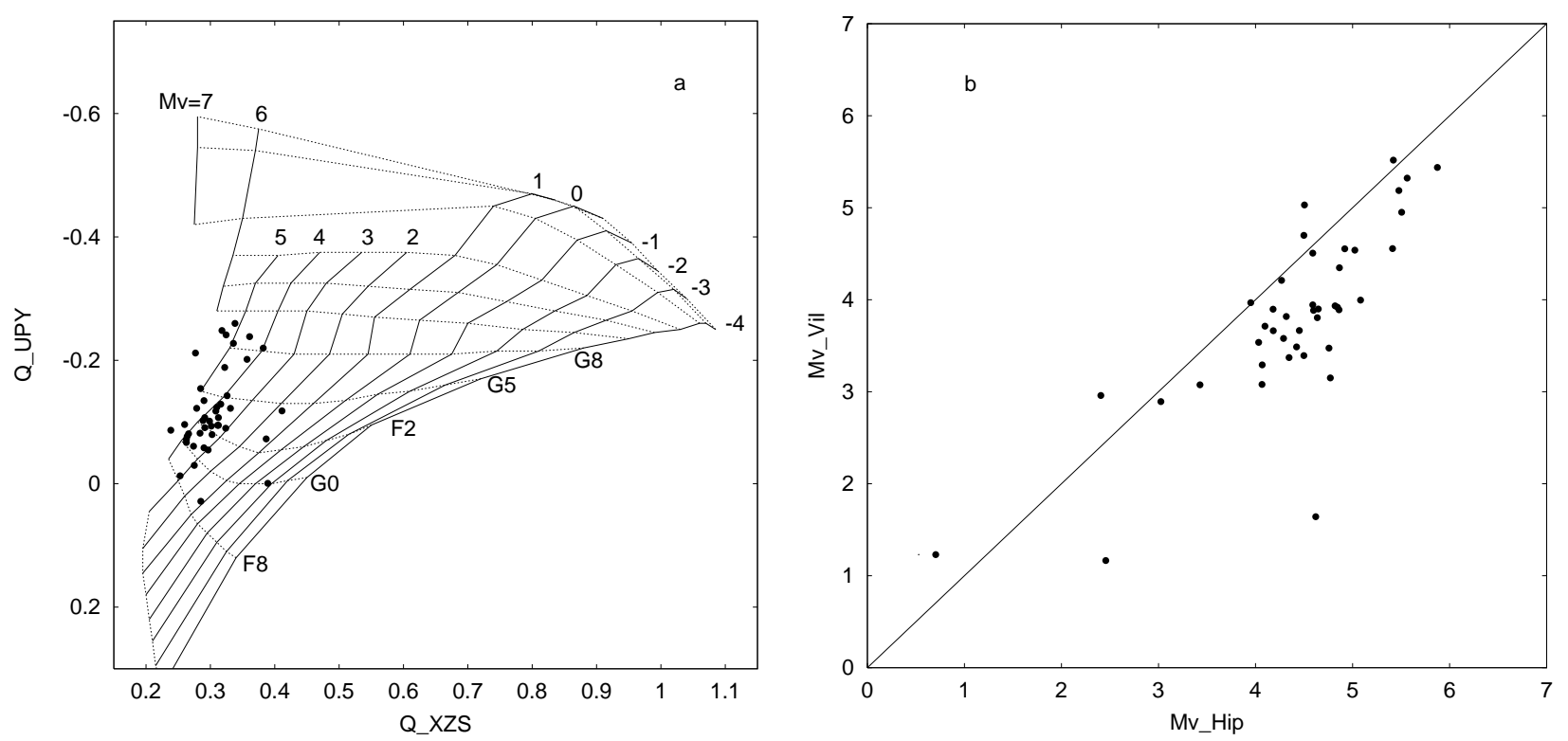

Fig. 8. G-type dwarfs. a) $Q Q$ classification diagram. b) The comparison between $M_{\mathrm{V}} \mathrm{HIP}$ and $M_{\mathrm{V}}$ VIL. The solid line is the 45 degree line.

\subsection{Possible influence of the Ca II H and $\mathrm{K}$ lines on deriving of $M_{V}$ via the Vilnius classification diagrams}

One possibility to obtain absolute magnitude $M_{\mathrm{V}}$ in the Vilnius system is via the classification $Q Q$ diagrams. If $X$ magnitude is influenced by the presence of stellar CaII lines, this may influence the position of the stars in the $Q Q$ diagrams, thus the inferred $M_{\mathrm{V}}$ values. The effect should be most pronounced for G-type dwarfs, since the decrease of the temperature toward K-dwarfs weakened the Ca II lines. Figure 8a presents the classification diagram $Q_{\mathrm{UPY}}$ vs. $Q_{\mathrm{XZS}}$, which is used for luminosity classification of G-type stars. The calibration grids are these from Straizys et al. (1982). A sample of G-type dwarfs is plotted as well, which consists of all G dwarfs from our G-type sub-sample with Hipparcos data available. $E_{\mathrm{UP}} / E_{\mathrm{PY}}=0.38$ and $E_{\mathrm{XZ}} / E_{\mathrm{ZS}}=1.13$ (Straizys 1977) were used to calculate $Q_{\mathrm{UPY}}$ and $Q_{\mathrm{XZS}}$. In this diagram a CaII effect should move a star almost perpendicular to the absolute magnitude $M_{\mathrm{V}}$ isolines, toward the negative side of $M_{\mathrm{V}}$ scale. Because of this we should expect the $M_{V}$ estimates for G-type dwarfs, obtained via $Q_{\mathrm{UPY}}$ vs. $Q_{\mathrm{XZS}}$ diagram to be more luminous in comparison to the true luminosities. The comparison of the $M_{\mathrm{V}}$ values obtained from the $Q_{\mathrm{UPY}}$ vs. $Q_{\mathrm{XZS}}$ diagram to the $M_{\mathrm{V}}$ values inferred from Hipparcos is shown in Fig. 8b. Indeed, the $Q Q$ method leads to brighter $M_{\mathrm{V}}$ than the Hipparcos estimates do, suggesting that for G-type stars the stellar Ca II lines influence in $X$ pass-band should be taken into account.

\section{Conclusions}

We present a number of empirically derived relationships between similar colour indices in the Vilnius and
Strömgren systems. In a comparison of all published Vilnius and Strömgren photometry we noticed significant differences in similar indices, apparently due to small deviations of central wavelength and band width. Deviations are most pronounced for the differently defined ultraviolet $(u$ and $U$ ) and violet $(v$ and $X$ ) bands. We suggest that the shift and widening of the Vilnius bands introduce a metal and luminosity sensitivity not present to the same degree in the Strömgren bands.

For a sample of $\mathrm{G}$ and $\mathrm{K}$ stars of moderate luminosity we show that the difference $(v-X)$ depends of the EQW of the stellar Ca II T lines, suggesting that for this spectral range the effect may be significant. We show that a clear correlation exists between $(v-X)$ and $h k$ index, which support the above statement. We also suggest that the presence of Ca II lines may effect the derivation of luminosity based on the classification $Q Q$ diagrams in the Vilnius system. The effect should be most significant for G-type dwarfs, leading to intrinsic luminosity estimates of about 0.7 mag brighter than the Hipparcos estimates in the average for the investigated sample.

As far as the characteristics of the Strömvil system are concerned, our study shows that a band in the violet region should be strictly confined to lie between the CaII $(\mathrm{H} \epsilon)$ and $\mathrm{H} \gamma\left(\mathrm{CH}^{+}\right)$, in order to avoid the influence of the stellar CaII on the luminosity determination. So, unless the violet pass-band has to be very narrow, $\mathrm{H} \delta$ has to be included. For late type stars of moderate luminosity, the $(v-X)$ index may be used as a luminosity indicator with very modest scatter of approximately 0.2 in $\log g$, which is almost independent of the $[\mathrm{Fe} / \mathrm{H}]$ ratio and interstellar reddening. It turns out as well to be very straightforward to make an estimation of the $[\mathrm{Fe} / \mathrm{H}]$ index with an accuracy of 0.4 dex (for the sample collated), using the Vilnius $U$ and Strömgren $u$ filters. Collecting more 
complete sample, and especially Ca II spectra, would cast more light on the effects of metallicity and luminosity in the interrelationships studied.

Acknowledgements. This work was supported by NATO/Danish Research Council. This research has made use of the Simbad database, operated at CDS, Strasbourg, France.

\section{References}

Anthony-Twarog, B. J., Twarog, B., Laird, J. B., \& Payne, D. 1991, AJ, 101, 1902

ESA 1997, The Hipparcos and Tycho Catalogues, ESA SP1200, vol. 1-17

ESA-SCI 2000, GAIA, Composition, Formation and Evolution of the Galaxy, Concept and Technology Study Report

Jønch-Sørensen, H. 1995, A\&A, 298, 799

Mallik, S. V. 1994, A\&AS, 103, 279

Mallik, S. V. 1997, A\&AS, 124, 359
Matsushima, S. 1969, ApJ, 158, 1137

Mihalas, D. 1966, ApJS, 1

Olsen, E. H. 1983, A\&AS, 54, 55

Philip, A. D., \& Egret, D. 1980, A\&AS, 40, 199

Twarog, B. A., \& Anthony-Twarog, B. J. 1995, AJ, 109, 2828

Straizys, V. 1973, A\&A, 28, 349

Straizys, V. 1977, Multicolour stellar photometry (Mokslas Publ. House, Vilnius, Lithuania)

Straizys, V., Crawford, D. L., \& Davis Philip, A. G. 1996a, Baltic Astronomy, 5, 83

Straizys, V., Kazlauskas, A., Boyle, R. P., Vrba, F. J., \& Smriglio, F. 1996b, Baltic Astronomy, 5, 165

Straizys, V., Kuriliene, G., \& Jodinskiene, E. 1982, Vilniaus Astronomijos Observatorijos Biuletenis, 60, 3

Straizys, V., \& Sviderskiene, Z. 1972, Bull. Vilnius Obs., 35, 1 Straizys, V., \& Zdanavicius, K. 1970, Vilniaus Astronomijos Observatorijos Biuletenis, 29, 15

Strömgren, B. 1966, ARA\&A, 4, 433

Valiauga, G., Vansevicius, V., \& Straizys, V. 1996, Baltic Astron., 5, 523 